\title{
Mathematical Model for Freeze-Thaw Durability of Concrete
}

\author{
ZDENĚK P. BAŽANT ${ }^{*}$ \\ Center for Concrete and Geomaterials, Northwestern University. Evanston. Illinois 60208
}

JENN-CHUAN CHERN

Department of Civil Engineering, Taiwan National University, Taipei, Taiwan

ARNOLD M. ROSENBERG and JAMES M. GAIDIS ${ }^{\star}$

W. R. Grace and Company, Columbia, Maryland 21044

\begin{abstract}
Although the equations governing the individual basic physical processes involved in freezing and thawing of concrete are known, a mathematical model for this complex phenomenon is unavailable. Its formulation is attempted in the present study. Desorption and absorption isotherms for concrete below $0^{\circ} \mathrm{C}$ are constructed on the basis of isotherms for concrete above $0^{\circ} \mathrm{C}$, using pore size distribution functions. Water movement during freezing or thawing is described as a double diffusion process, involving both macroscopic diffusion through concrete and local diffusion of water into or out of air-entrained bubbles. Heat conduction is formulated taking into account the latent heat of freezing. Pore pressures are used in a two-phase material model, which makes it possible to predict the stress in the solid structure of concrete caused simultaneously by freezing and applied loads. This in principle reduces the freeze-thaw durability problem to the calculation of stresses and strains. However, development of the model to full application would require various new types of tests for calibration of the model, as well as development of a finite element code to solve the governing differential equations. Such a mathematical model could be used to assess the effect of cross-section size and shape, the effect of cooling rate, the delays due to diffusion of water and of heat, the effect of superimposed stresses due to applied loads, the role of pore size distribution, the role of permeability, and other factors which cannot be evaluated at present in a rational manner.
\end{abstract}

\section{Introduction}

$\mathbf{F}$ REEZE-THAW damage in concrete is a complex physical phenomenon about which much has been learned during the last 50 years. The Reference section lists the principal contributions. ${ }^{1-24}$ Essential results were obtained by Powers ${ }^{18.19}$ and Fagerlund. ${ }^{48}$

Powers' hydraulic pressure theory of freeze-thaw damage established the central notion that damage is caused by pore pressures induced by the expulsion of water on freezing. This theory shows that the pore pressures in concrete can be greatly reduced by providing additional empty pores into which water can be expelled from the capillary pores in the hardened cement paste as the water contained in them freezes. Based on this concept, the current practice of protection against freeze-thaw damage is the use of air-entraining agents, which create these additional pores, i.e., air bubbles. It also follows from Powers' hydraulic pressure theory that the air bubbles must be very small so that their spacing can be so small that the transport of water from the freezing \footnotetext{
Manuscript No. 199743. Received December 2. 1986: approved March 25. 1988.
Presented at the 88th Annual Meeting of the American Ceramic Society. Chicago, IL. April 28. 1986 (Cements Division. Paper No. 12-T-86).

Member, the American Ceramic Society.
}

capillary pores into the bubbles does not require development of high pore pressures. The hydraulic pressure theory was later extended and modified in Helmuth' $\mathrm{s}^{10}$ osmotic pressure theory.

Powers showed that the water in saturated large capillaries was responsible for the damage due to freezing and thawing. This fact was emphasized and demonstrated more clearly in the work of Fagerlund. ${ }^{4-8}$ If the large pores are empty, concrete is safe from freeze-thaw damage even if no air entrainment is used. This occurs when concrete is only partially saturated, with water content being below approximately $90 \%$ of the water content at total saturation.

Reduction of water content below the critical degree of saturation. however, cannot be easily exploited for freeze-thaw protection of concrete structures. Drying below the critical limit takes an unacceptably long time for typical structure dimensions (thickness) and is even undesirable because it leads to cracking and arrests the hydration process. Likewise, use of air entrainment is not an ideal solution. The creation of additional pores, typically about $6 \%$ of the volume of concrete, causes a reduction of the strength and fracture toughness of concrete. Moreover, many other factors influence freeze-thaw damage. For example, for a certain cross-section size, cooling rate, pore size distribution, etc., the use of a $6 \%$ air entrainment might be necessary while for another cross-section size, cooling rate, or pore size distribution, a much smaller air entrainment (or no air entrainment) might suffice.

The rate of expulsion of water from the freezing capillary pores into the air-filled pores depends on the cooling rate. The cooling rates are very different in the interiors of thin and thick cross sections because of the delay caused by heat conduction. Furthermore, susceptibility to freeze-thaw damage must obviously depend on the permeability of concrete, not only on the permeability for the microscopic flow between small and larger pores, but also on the permeability and diffusivity for macroscopic water movements through concrete. The capability of concrete to reduce pore pressures by macroscopic diffusion depends on the size and shape of the cross section of the structure.

All these phenomena are at least partially understood at present, but only in a qualitative sense. However, a practical evaluation of these numerous influences cannot be done without a mathematical model, since pore pressures produced by the freezing process must be compared with the strength of the material. The purpose of the present study (based on a manuscript privately communicated to Rosenberg on June 8,1982 ) is to formulate a comprehensive mathematical model of these phenomena, to serve as a basis for further development and guide in experimental studies. The emphasis of this study will be on the role of diffusion phenomena coupled with the effect of pore size distribution.

Most of the factors to be analyzed here have been qualitatively known for a long time. Why is it only today that a comprehensive mathematical model is attempted? Application of such a model requires a computer, and it is only because of computers that development of a mathematical model makes sense and ought to be undertaken. 


\section{Sorption Isotherms}

Essential properties for the analysis of diffusion are the relations between the specific water content of concrete, $w$, and pore pressure, $p$, at various temperatures and at states of thermodynamic equilibrium among various phases of water in the pores. ${ }^{25-28}$ The typical desorption isotherms for various temperatures above the freezing point of water are illustrated in Fig. $l(A)$, in which $w_{1}$ is the water content of concrete at saturation and $p_{s}$ is the vapor pressure at saturation, which is a function of temperature $T$. For partially saturated states, $p<p_{s}$, pressure $p$ is understood as the pressure of water vapor, which is different from the pressure of capillary water.

The total specific water content of pores, $w_{p}$, may be subdivided into two categories: the gel water $\left(w_{g}\right)$, which is the water in the pores of cement gel, having a width $<30 \AA$ ( $<3.0 \mathrm{~nm})$; and capillary water $\left(w_{c}\right)$, which fills the larger pores. ${ }^{29}$ The capillary water may further be subdivided into water in the small capillary pores $\left(w_{c 1}\right)$, which are less than about $1300 \AA(130 \mathrm{~nm})$ in width, and water in the large capillary pores $\left(w_{c 2}\right)$, which are up to about $5000 \AA(500 \mathrm{~nm})$ in width. The latter appears from experience to be important for freeze-thaw damage. In the case of air entrainment, there also exist bubbles of typical diameter $25 \mu \mathrm{m}$, and when these get filled by water, their water content is denoted as $w_{b}$. Thus, the subdivision of the total specific water content may be written as

$$
\begin{aligned}
& w=w_{p}+w_{b} \\
& w_{p}=w_{g}+w_{c} \\
& w_{c}=w_{c 1}+w_{c 2}
\end{aligned}
$$

The various components of pore water are graphically shown in Fig. $1(A)$.

The isotherms for absorption always lie significantly below the isotherms for desorption, as illustrated in Fig. $1(B)$. When pore pressure $p$ is controlled and its decrease is reversed to an increase, $w$ remains almost constant until the increasing pore pressure reaches the absorption isotherm, and on the other hand, when an increasing pore pressure is reversed to a decrease, $w$ again remains almost constant until the desorption isotherm is reached. This phenomenon represents a hysteresis of sorption cycles and is important when pore water diffusion in a cyclic environment is to be calculated.

The pore structure may be characterized by the distribution $\phi(r)$ of the volumes of the pores of various widths $2 w ; \phi(r) d r$ is defined as the volume of all the pores in a unit volume of concrete whose widths lie between $r$ and $r+d r$. The total specific water content of saturated concrete may be expressed as

$$
w=\int_{r_{\min }}^{r_{\max }} \beta \rho_{w} \phi(r) d r
$$

in which $\rho_{w}=1 \mathrm{~g} / \mathrm{cm}^{3}$ is the mass density of water at $1 \mathrm{~atm}$ and $4^{\circ} \mathrm{C}$, and $\beta$ is the coefficient representing the degree of saturation of the pores of width $2 r ; r_{\min }$ and $r_{\max }$ represent the minimum and maximum half-widths of the pores in concrete.

For calculations of water diffusion above $0^{\circ} \mathrm{C}$, it is normally sufficient to characterize the sorption properties of concrete by the isotherms $w(p, T)$. For the analysis of freezing, it is convenient to refer the isotherms to pore sizes $r$. This makes it possible to determine the change of water content of pores of size $r$ from the known change of mass density of water due to freezing. Since calculations are next to impossible for pores of arbitrary geometry, the pores are usually imagined as cylindrical, in which case the capillary meniscus in the pore is spherical, of radius $r$. If such a pore is partially saturated by water, the condition of equilibrium of the surface tensions on the meniscus with the pressure, $p_{c}$, in the capillary (liquid) water (which is negative, i.e. tension) yields the well-known Laplace equation $p_{\mathrm{c}}=-2 \gamma(T) / r$, in which $\gamma(T)$ is the surface tension of liquid water at temperature $T$ (about $75 \mathrm{dyn} / \mathrm{cm}\left(7.5 \times 10^{-2} \mathrm{~N} / \mathrm{m}\right)$ at $\left.25^{\circ} \mathrm{C}\right)$.

The pressure in capillary water may also be calculated from the
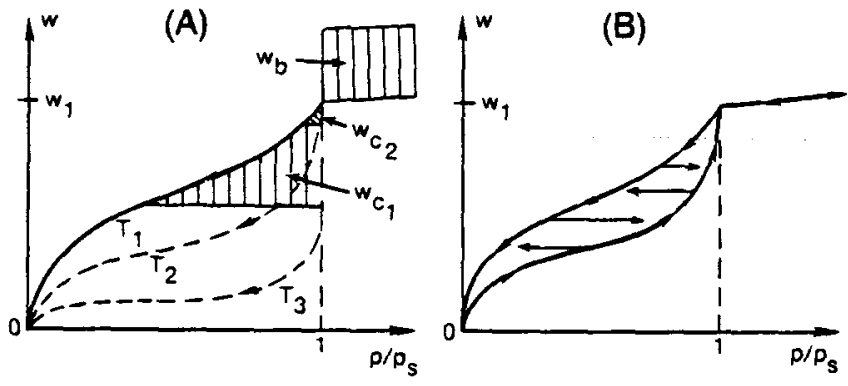

Fig. 1. Typical shapes of desorption and absorption isotherms of concrete and distinction between various types of water in the pores.

condition of equal chemical potentials (Gibbs free energy per unit mass) in the liquid water and vapor, treating water vapor approximately as an ideal gas. This yields the Kelvin equation, $p_{c}=\rho_{w}(R T / M) \ln h, h=p / p_{s}(T)$, in which $h$ is the pore relative humidity, $R$ the universal gas constant, and $M$ the molecular weight of water, i.e., $18.02 \mathrm{~g} / \mathrm{mol}(R=82.06 \mathrm{~atm} /(\mathrm{K} \cdot \mathrm{mol}))$. Combining the Laplace and Kelvin equations, one obtains the following well-known equation for pressure $p_{0}$ at which a cylindrical pore of radius $r$, at temperature $T$, gets filled:

$$
p_{0}(r, T)=p_{s}(T) \exp \left(-\frac{2 \gamma(T) M}{R T \rho_{w} r}\right) \quad(\text { for } T>273 \mathrm{~K})
$$

Consider now the freezing of pore water. The freezing point of capillary water is depressed below $0^{\circ} \mathrm{C}$, depending on pore size $r$. This depression may be calculated by considering the change of the difference in the chemical potentials $\Delta \mu$ between ice and liquid water. Initially, $\Delta \mu$ must be equal to zero at $0^{\circ} \mathrm{C}$. During freezing we must have $d(\Delta \mu)=v_{i} d p-\Delta S d T=0$, where $\Delta S=-C_{i} / T_{0}$ is the difference in entropy between ice and liquid water, $T_{0}$ is the normal freezing temperature $(273 \mathrm{~K})$, and $C_{i}$ is the latent heat of fusion of water (liquid to ice). ${ }^{25-28}$ Substituting and integrating, we get $v_{i} p=-\left(C_{i} / T_{0}\right)\left(T_{0}-T_{f}\right)$. For pressure $p$ in ice we may now, as an approximation, introduce the Laplace equation $p=2 \gamma_{i} / r$ where $r$ is the radius of a cylindrical pore and $\gamma_{i}$ is the surface tension of ice (approximately $20 \mathrm{dyn} / \mathrm{cm}$ $\left.\left(2.0 \times 10^{-2} \mathrm{~N} / \mathrm{m}\right)\right)$. Substitution into the preceding equation then yields Kubelka's equation:

$$
T_{f}=T_{0}\left(1-\frac{2 \gamma_{i} v_{m}}{C_{i} r}\right)
$$

For pores of radii $r=500,100,50,20$, and $10 \AA(50,10,5,2$, and $1 \mathrm{~nm}$ ), this equation yields the freezing point depressions $T_{f}-T_{0}=-0.67,-3.3,-6.7,-16.8$, and $-33.5 \mathrm{~K}$, respectively.

Because of simplifications in derivation, such as the consideration of a cylindrical pore and a spherical meniscus only, and the neglect of shear stiffness of ice, etc., this equation is, of course, only approximate; but it seems to agree reasonably well with some measurements. A more serious deviation nevertheless occurs in practice: the presence of solutes in water causes an appreciable further depression of the freezing point, which can be calculated if the ion concentrations are known. ${ }^{29}$ However, to take this into account, we would need to introduce as further variables the concentrations of various ions in the pore water of concrete. To avoid such formidable complications we neglect this effect. This seems acceptable if the concentrations of solutes in pore water are approximately constant, since Eq. (4) would still approximately hold, although with a larger value of $\gamma_{i}$.

In concrete that is fully saturated, which occurs when $p \geq$ $p_{s}(T)$, the ice may be assumed to behave as a bulk phase. By application of a sufficiently large pressure, ice may be caused to melt (same as any crystal whose volume decreases on melting). The melting pressure, identical with the crystal pressure, may be 
(A)

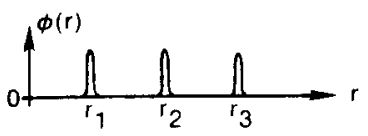

Cumulative

(B)
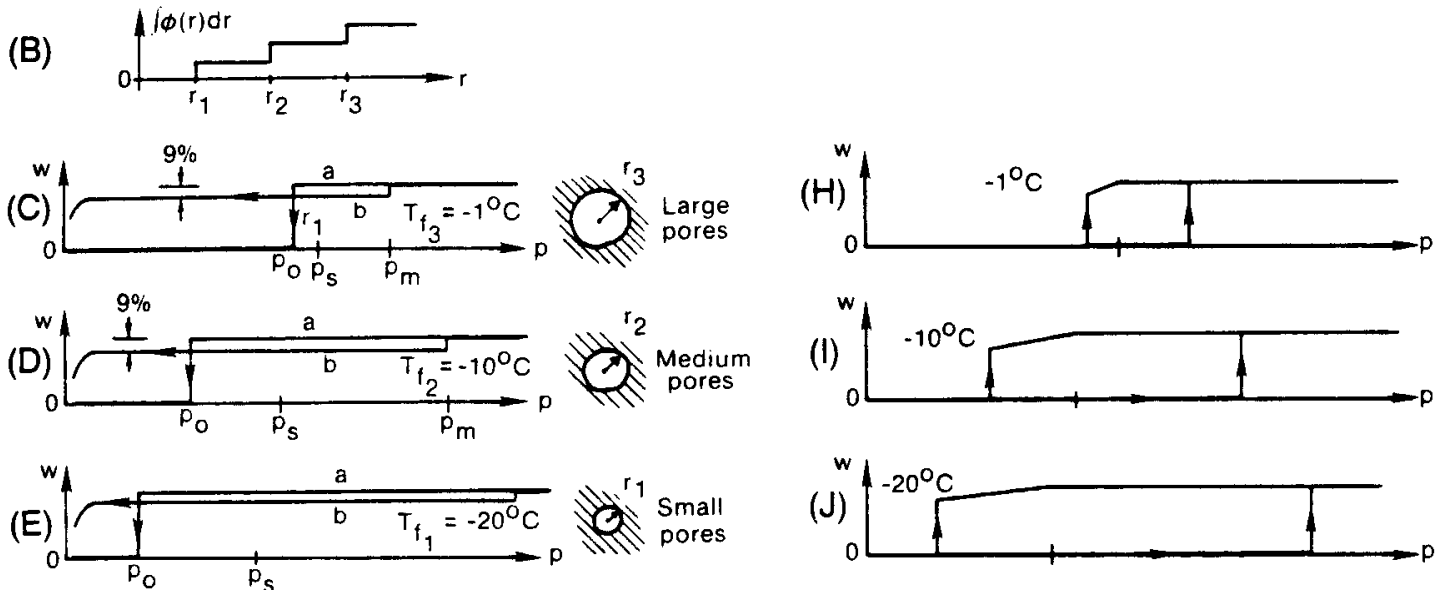

$\downarrow$
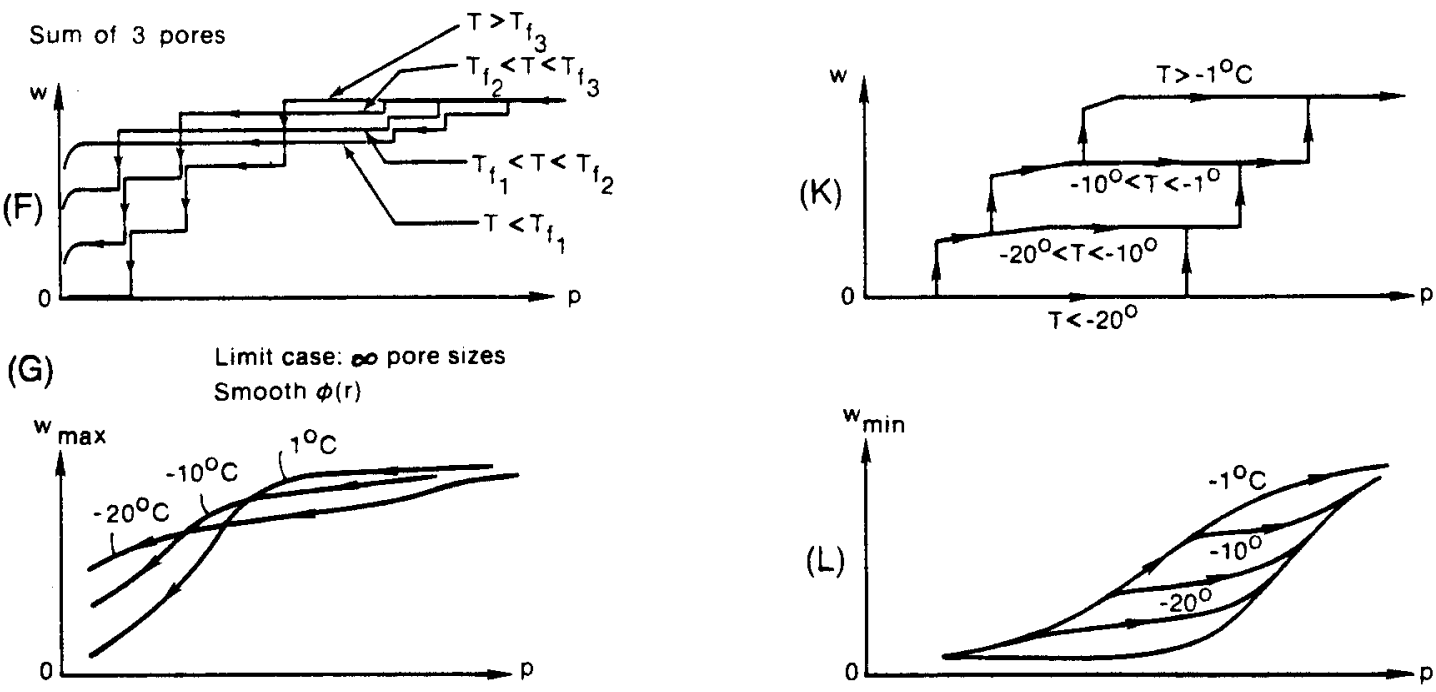

Fig. 2. Construction of desorption and absorption isotherms for concrete below $0^{\circ} \mathrm{C}$.

approximately calculated from the equation ${ }^{18}$

$$
p_{m}=1+93.1\left(T_{0}-T\right) \quad\left(\text { for } T<T_{0}\right)
$$

in which the pressure is in atmospheres $(0.10132 \mathrm{MPa})$ and the temperature in kelvins. Again, the presence of solutes no doubt changes the coefficient of this equation. This would require interpreting $p_{m}$ as the osmotic pressure of ice, which would complicate the formulation. However, this effect is probably small and we neglect it.

To understand how freezing affects the sorption isotherms, consider first a solid with only three different pore sizes $r_{1}, r_{2}, r_{3}$, for which the pore volume distribution function consists of three Dirac delta functions as sketched in Fig. 2(A). The cumulative pore volume distribution then consists of three steps, as sketched in Fig. 2(B). For the moment, assume that the pores are precisely cylindrical, in which case there can be no difference between the desorption and absorption isotherms. Figures $2(C),(D)$, and $(E)$ show the curves of desorption isotherms for these pore sizes taken individually. Water fills or empties the pore at pressure $p_{0}$ indicated by Eq. (3) for the corresponding pore radius $r_{1}$ or $r_{2}$ or $r_{3}$, and so the corresponding isotherm for each individual pore is a step function. Upon freezing, the specific volume of water increases by about $9 \%$. Therefore, if this capillary pore is filled, its water content must decrease by about $9 \%$ as shown by curves $b$ in Figs. $2(C)$ to $(E)$. However, this expulsion of water terminates at the melting point of ice with the corresponding pressure, $p_{m}$ (see Figs. $2(C)$ to $(E)$ ). Note that for different pore sizes the steps due to melting in Figs. $2(C)$ to $(E)$ occur at different temperatures, $T_{f 1}, T_{f 2}$, and $T_{f 3}$.

The behavior of the system of the three pore sizes is now obtained by summing the $w$ values of desorption isotherms for the three individual pore sizes (Figs. $2(C)$ to $(E)$ ). This has to be done separately for four temperature ranges below $T_{f 1}$, between $T_{f 1}$ and $T_{f 2}$, between $T_{f 2}$ and $T_{f 3}$, and above $T_{f 3}$. The desorption isotherms obtained by this summation are shown in Fig. $2(F)$.

A similar construction may be imagined for a system of many discrete pore sizes, in which case Fig. $2(F)$ would involve many isotherms with many small steps and many different pressure values. In the limit of infinitely many pore sizes, i.e., for a continuous pore size distribution, one would obtain curves of the type shown in Fig. $2(G)$. It is mathematically inconvenient that these isotherms for various temperatures mutually intersect; i.e., the same water content may be obtained for the same pressure at different temperatures, which is impossible above $0^{\circ} \mathrm{C}$.

The foregoing construction applies only for desorption isotherms starting from a fully saturated concrete. Because the pores 
are not cylindrical, it is possible that the same total Gaussian curvature of the meniscus is obtained for various geometric forms of the liquid-vapor interface, with the consequence that, for a given pressure, equilibrium is possible for a certain range of water contents.

A similar phenomenon probably occurs at very low humidities, in which case the water content is varied in the gel pores. The reason is that, because of complexity of the pore shape, different geometrical forms and different thicknesses of the adsorption layers on the walls of gell pores can be in equilibrium at the same pore pressure.

The maximum possible water content occurs for each pressure during desorption from an initially saturated state. On the other hand, the minimum possible water content for each pressure occurs during absorption from an initially fully dried state (Fig. $1(B)$ ). During arbitrary positive and negative variations of pore pressure, intermediate water contents between the maximum $w_{\max }$ and the minimum $w_{\min }$ are obtained (Fig. $1(B)$ ). On the other hand, for pressures above the saturation pressure, $p_{s}(T)$, only one water content can correspond to each given pressure, as capillary phenomena do not take place.

Consider now the isotherms for absorption from a fully dried state. They give for each pressure $p$ the minimum possible water content, $w_{\max }$. For the system of three discrete pore sizes, the pore of each size gets filled by water when pressure $p_{0}$ given by Eq. (3) is reached. However, if the pore is not cylindrical but has a complicated shape, then water fills this pore only to a certain fraction $1-\psi$ of its maximum possible water content, where $\psi<1$. This incomplete filling of the pore must terminate when $p$ reaches the value of $p_{s}(T)$, at which the pore becomes completely filled. This is indicated by the inclined rising portion of the curves above the freezing temperatures, $T_{f i}$, in Figs. $2(H)$ to $(J)$. When the temperature is below the freezing temperature for the given pore size, the pore does not fill at pressure $p_{0}$ given by Eq. (3), but can remain empty of ice until the melting pressure for $p_{m}(T)$ of ice for a given temperature is reached. The pore must fill completely before the pressure can be raised further. This is indicated by the second rising vertical segments in Figs. $2(H)$ to $(J)$.

By summing the ordinates from Figs. $2(H)$ to $(J)$ for the pores of threee different sizes, one obtains the four isotherms for four temperature intervals as shown in Fig. $2(K)$. In the limit of infinitely many continuously distributed pore sizes, one would obtain in this manner the isotherms for absorption which have the shape of the curves shown in Fig. 2(L). They represent the minimum possible water contents for various pressures at various temperatures. For arbitrary changes of pressure, the following rules apply:

(1) If the state $w(p, T)$ is on the desorption isotherm $\left(w_{\max }\right)$, and if $d p<0$, the local desorption isotherm (Fig. $2(G)$ ) is followed. However, if at the same time $d p>0, w$ will remain almost constant, although for numerical calculation it is convenient to prescribe a very small but finite slope $k=d w / d p$. If the current state is between the desorption and absorption isotherms, i.e., $w_{\min }<w_{\max }$, pressure change leads to almost no change in $w$ (i.e., to a very small slope $k$ ), regardless of the sign of $d p$.

(2) If the current state is on the absorption isotherm $\left(w_{\min }\right)$, and if $d p>0$, the local absorption isotherm (Fig. $2(L)$ ) is followed. However, if $d p<0$, again the water content remains almost constant at decreasing pressure.

The behavior between the pair of curves for the same temperature shown in Figs. $2(F)$ and $(L)$ is similar to that already illustrated in Fig. $l(B)$ for a pair of simple absorption and desorption isotherms.

Finally, if temperature $T$ changes, the state points $w(p, T)$ cross horizontally (at $w=$ constant) from one isotherm to another; i.e., a temperature change is accompanied by a pressure change without any change in the specific water content. A simultaneous increment of pressure and temperature may be treated as a sequence of small increments of pressure at constant temperature, followed by small increments of temperature at constant pressure. etc
The desorption-absorption behavior that we have just described may be conveniently characterized in terms of coefficient $\beta$ in Eq. (2), which is considered as a function of pore radius $r$, pressure $p$, and temperature $T$. Based on the preceding analysis, the following rules for coefficient $\beta$ can be formulated:

(1) For $T>T_{f}(r)$

$$
\begin{aligned}
& \left.\begin{array}{l}
\beta=1 \quad \text { for } p>p_{s}(T) \\
\beta_{\max }=1 \\
\beta_{\min }=1-\psi
\end{array}\right\} \text { for } p_{0}(r, T)<p<p_{s}(T) \\
& \begin{array}{l}
\beta=0 \quad \text { for } p<p_{0}(r, T)
\end{array}
\end{aligned}
$$

(2) For $T<T_{f}(r)$

$$
\left.\begin{array}{l}
\beta=1 \quad \text { for } p>p_{m}(T) \\
\beta_{\text {max }}=0.91 \\
\beta_{\text {min }}=0
\end{array}\right\} \text { for } p<p_{m}(T)
$$

The value of $\psi$ cannot be calculated and has to be calibrated on the basis of measurements of hysteresis cycles. From some typical isotherms, it is found that $\psi=0.1$ to 0.3 . The values of $\beta_{\max }$ and $\beta_{\min }$ apply for the desorption isotherm $w_{\max }$ (Fig. $2(G)$ ) and for the absorption isotherm $w_{\min }$ (Fig. $2(L)$ ), respectively. The correctness of Eqs. $(6 a)$ to $(6 e)$ is most easily verified for the special case of three or fewer discrete pore sizes.

Equations $(6 a)$ to $(6 e)$ define only the isotherms of $w_{\max }$ and $w_{\min }$. The intermediate behavior, at which the value of $w$ is almost constant at variable $p$ or $T$, may be described, according to the preceding discussion, by the following equations:

$$
\begin{aligned}
& \text { If } \beta^{(i)}=\beta_{\max } \text { and } \Delta w_{\max }(p, T)<0 \\
& \beta^{(i+1)}=\beta_{\max } \\
& \text { If } \beta^{(i)}=\beta_{\max } \text { and } \Delta w_{\max }(p, T) \geq 0 \\
& \Delta w=\nu_{0} \Delta p \\
& \text { If } \beta^{(i)}=\beta_{\min } \text { and } \Delta w_{\min }(p, T)>0 \\
& \Delta w=\nu_{0} \Delta p \\
& \text { If } \beta^{(i)}=\beta_{\min } \text { and } \Delta w_{\min }(p, T) \leq 0 \\
& \beta^{(i+1)}=\beta_{\min } \\
& \text { If } \beta_{\min }<\beta^{(i)}<\beta_{\max } \\
& \Delta w=\nu_{0} \Delta p
\end{aligned}
$$

in which superscript $(i)$ denotes the initial state before a small increment $\Delta p, \Delta T, \Delta w$, and superscript $(i+1)$ labels the final state after this increment. Coefficient $\nu_{0}$ is approximately 0 , but for convenience of numerical computations it should be taken as a small positive value, perhaps 0.01 of the mean slope of the sorption isotherm at room temperature.

For the description of sorption isotherms above $0^{\circ} \mathrm{C}$, the use of the pore volume distribution function $\phi(r)$ is not necessary.

However, there is an advantage in using this function in the case of freezing. The advantage consists in the possibility of applying to the volume of pores of each different size the $9 \%$ mass density change of water on freezing, and then distinguishing among the freezing temperatures for different pore sizes. In this manner, we are able to construct the sorption isotherms for freezing on the basis of the isotherms above $0^{\circ} \mathrm{C}$, although experimental calibration of the coefficients for frozen concrete might be desirable, given that our theoretical formulation is not without error.

The fact that we associate functions $\phi(r)$ (at $\beta=1$ ) with the desorption rather than absorption isotherm is arbitrary. Although for the sake of practicability we have to treat the pore spaces as cylindrical, function $\phi(r)$ represents the equivalent pore volumes if the capillaries were cylindrical, and not the exact volumes of the pores of width $2 r$. But the error caused by this is probably 


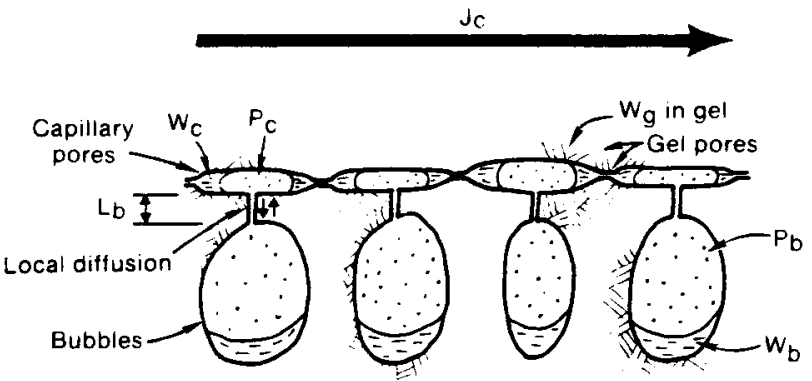

Fig. 3. Idealization of the pore system, with a subdivision into bubbles, gel pores, and capillary pores in the cement paste.

unimportant, because functions $\phi(r)$ cannot be calculated theoretically anyway, and must, in reality, be calibrated from some sort of sorption measurements. Although this function can be derived from measurements of adsorption of nitrogen or other gases, or by mercury porosimetry, we prefer to determine this function from water sorption tests because we apply it to water.

By definition (Eq. (2)), function $\phi(r)$ may be expressed as $\rho_{w} \phi(r)=d w / d r=(d w / d p)(d p / d r)$. Here we may substitute $d w / d p=(d w / d h) / p_{s}(T)$ where $h=p / p_{s}(T)$, and $d p / d r=$ $p_{s}\left(A_{T} / r^{2}\right) \exp \left(-A_{T} / r\right)$, in which $A_{T}=2 \gamma(T) M /\left(R T \rho_{w}\right)$. Thus we obtain the equation

$$
\rho_{n} \phi(r)=\frac{A_{T}}{r^{2}} e^{-A T^{\prime}} \frac{d w^{\prime}}{d h}
$$

which makes it possible to calculate the specific pore volume distribution function from the measured slopes $k=d w / d h$ of the desorption isotherms.

The mass density $\rho_{n}$ of water in Eqs. (8) and (3) may be considered for the case of partially saturated concrete $\left(p<p_{s}\right)$ to be approximately a constant, equal to the mass density of water at $0^{\circ} \mathrm{C}$ and $p=1 \mathrm{~atm}$. This means that the changes of mass density of liquid capillary water due to cooling below $0^{\circ} \mathrm{C}$ are neglected. and so is the mass of vapor in the pores. For saturated concrete, however. the changes of mass density of water do not have a negligible effect, and therefore $\rho_{n}$ for $p>p_{\text {s }}$ should be taken into account, and may be evaluated from the well-known equation of state of water (see, e.g.. ASTM Steam Tables and Ref. 28).

\section{Water Diffusion}

In early studies, three periods of the drying process were distinguished: initial, funicular, and pendular. For each of them a different set of moisture-transfer relations was formulated, distinguishing between transfers of liquid water and of vapor. However, these classical distinctions now appear to be unnecessary complications and at the same time not quite realistic.

The distinctions between the various drying periods were necessary since the differential equations were assumed to be linear. Today there is no problem in solving nonlinear diffusion equations numerically with a computer, and a single diffusion equation with coefficients dependent on pore humidity (or water content) can describe the entire process.

Equations for the final pendular state were based on the assumption that moisture transfer takes place in the form of vapor. This assumption is questionable, for two reasons: (1) The capillaries in hardened cement paste of good quality are not continuous, as established by Powers, Copeland, and Mann ${ }^{31}$, and (2) vaporized water molecules cannot pass through the molecule-sized pores of the cement gel, because the mean free path of a vaporized water molecule exceeds by several orders of magnitude the widths of these pores (see also Ref. 32). Furthermore, since local thermodynamic equilibrium between various phases of water is assumed to exist in all diffusion models, the vapor pressure, the pressure in capillary liquid water, the surface tension in adsorbed water layers, and the moisture content are all functionally related. Con- sequently, the gradient of one is proportional to the gradient of any other, with the proportionality coefficient being a function of these quantities.

Thus, the moisture transfer can be characterized by a single nonlinear diffusion equation in terms of any of the aforementioned variables. The choice can be made for mathematical convenience. Bažant and Thonguthai showed that the choice of pressure $p$, representing the vapor pressure for unsaturated concrete and the pore water pressure for saturated concrete, is a convenient choice since grad $p$ then gives the water flux not only at uniform temperature but also in the presence of temperature gradients (because $p$ varies with $T$ ). The governing equation for the mass flux $J$ of water $\left(\mathrm{kg} /\left(\mathrm{m}^{2} \cdot \mathrm{s}\right)\right)$ is expressed in terms of Darcy's law:

$$
\vec{J}=-a \operatorname{grad} p
$$

in which $a=a(w, T)$ is the permeability, which depends on $p$ or $\boldsymbol{w}$, as well as $T$. The approximate form of this dependence has been established. ${ }^{33-36}$

To predict the water movement into and from the bubbles (Fig. 3), the water content $w_{b}$ of the bubbles must be introduced as a separate quantity. distinct from the water content $w$ of concrete. bubbles excluded. The conservation of the mass of water then requires

$$
\operatorname{div} \vec{J}=-\dot{\boldsymbol{u}}, \dot{\boldsymbol{w}}_{b}
$$

where superimposed dots denote partial time derivatives. The diffusion of water into or out of the bubbles may be characterized by the dependence of the rate of the water content in the bubbles, $\dot{w}_{b}$. on the pressure difference between the pores of hardened cement paste and the bubbles

$$
\dot{w}_{b}=\frac{c}{L_{b}}\left(p-p_{b}\right)
$$

in which $c$ is the appropriate permeability coefficient and $L$ is the mean distance of passage of water molecules between the bubbles and the pores of the surrounding cement paste. One may assume that, approximately, $c=a$.

The distance $L_{b}$ may be estimated from the volume fraction of bubbles, $v_{b}$, and the mean radius of the bubbles, $r_{b}$. Assuming the bubbles to form a cubic array with spacing $s_{b}$, the number of bubbles per unit volume is $N=1 / s_{b}^{3}$. Since $v_{b}=4 \pi r_{b}^{3} N / 3$, and the distance $L$ may be estimated as half the distance between the bubbles minus their radius, we have

$$
s_{b}=\left(\frac{3 v_{b}}{4 \pi}\right)^{1 / 3} \frac{1}{r_{b}}, \quad L_{b}=\frac{s_{b}}{2}-r_{b}
$$

The sorption relation for the water content of the bubbles has the form $p_{b}=p_{b}\left(w_{b}, T\right)$, and its form may be easily derived by considering a spherical meniscus in a spherical bubble pore, for the case of unfrozen concrete. The rate of pressure change in the bubbles may then be expressed as

$$
\dot{p}_{b}=\frac{\partial p_{b}}{\partial w_{b}} \dot{w}_{b}+\frac{\partial p_{b}}{\partial T} \dot{T}
$$

After substituting Eq. (11), we then have

$$
\dot{p}_{b}=\frac{p-p_{b}}{\tau_{b}}-c_{b} \dot{T}
$$

in which we introduce the notation

$$
\tau_{b}=\frac{c}{L_{b}}\left(\frac{\partial p_{b}}{\partial w_{b}}\right)_{T}, \quad c_{b}=\left(\frac{\partial p_{b}}{\partial T}\right)_{w_{b}}
$$

$\tau_{b}$, a constant having the dimension of time, may be regarded as the effective halftime for the diffusion between the bubbles and the surrounding hardened cement paste; $c_{b} \dot{T}$ is the rate of change of bubble pressure caused by temperature change.

Substituting Eq. (9) into Eq. (10), we have for the macroscopic 
diffusion through concrete the equation

$$
\dot{w}+\dot{w}_{b}=\operatorname{div}(a \operatorname{grad} p)
$$

Considering the sorption isotherms for concrete without bubbles in the form $p=p(w, T)$, we have

$$
\dot{p}=\frac{\partial p}{\partial w} \dot{w}+\frac{\partial p}{\partial T} \dot{T}
$$

Eliminating $\dot{w}$ from this equation and Eq. (16), we then obtain the final governing equation for the macroscopic diffusion through concrete:

$$
\dot{p}=k \operatorname{div}(a \operatorname{grad} p)-k \dot{w}_{b}+c_{T} \dot{T}
$$

in which we have introduced the notation

$$
k=\left(\frac{\partial p}{\partial w}\right)_{T}, \quad c_{T}=\left(\frac{\partial p}{\partial T}\right)_{w}
$$

Here $k$ represents the slope of the desorption or absorption isotherm (depending on the sign of $\dot{p}$ ). Equation (18) can be solved by the Galerkin-type finite-element method with the Crank-Nicolson time-step algorithm. ${ }^{33-35}$ Simplified analytical solutions may be obtained according to linear diffusion theory if coefficients $k$ and $a$ are considered as constant, having the average values for the range under consideration. The diffusion equation for the flow into or out of the bubbles (Eq. (11)) is an ordinary differential equation, easily solved by a step-bystep algorithm.

For the treatment of freezing, it is of interest to capture the delay in adjustments of pore pressures due to the time needed for water molecules to flow between the pores of cement paste and the air-entrainment bubbles (Fig. 3). This flow is local in character, since the bubbles are not continuous. Because of this fact, and also because the water molecules move across the bubbles much faster than across the cement paste, the rate of macroscopic diffusion is doubtless controlled mainly by the cement paste. Therefore, the presence of the bubbles must have only a negligible effect on the macroscopic diffusion of water through concrete.

\section{Heat Transfer}

The sorption behavior with phase transitions of water introduces distributed heat sources or sinks. $\dot{H}_{1}, \dot{H}_{s}$, and $\dot{H}_{w}$. Thus, the differential equation for heat conduction takes in general the form

$$
\rho C \dot{T}=\operatorname{div}(b \operatorname{grad} T)-\dot{H}_{l}-\dot{H}_{s}-\dot{H}_{w}
$$

Here $\rho$ and $C$ are the mass density and the isobaric heat capacity of concrete (per unit mass of concrete. excluding free water), $\dot{H}_{l}$ is the heat supply rate due to the release of latent heat associated with freezing or thawing, $\dot{H}_{s}$ is the heat supply rate due to sorption heat, and $\dot{H}_{w}$ is the heat supply rate due to heat convection caused by the movement of water. The last two quantities are no doubt negligible, i.e., $\dot{H}_{s}=\dot{H}_{w}=0$. However. $\dot{H}_{l}$ probably has an appreciable effect in freezing-thawing problems. The latent heat is liberated on crossing the freezing or thawing temperature $T_{f}$ for a given pressure (Fig. 4). However. for numerical calculations it is convenient to assume that the latent heat is liberated gradually between temperatures $T_{f}-\Delta_{f}$ and $T_{f}+\Delta_{f}, \Delta_{f}$ being a small value such as $1 \mathrm{~K}$. Then the source term due to the latent heat may be written as

$$
\dot{H}_{l}=-\frac{C_{l}}{2 \Delta_{f}} w \dot{I}_{T}
$$

in which $C_{l}$ is the latent heat of freezing or thawing of water $(80 \mathrm{kcal} /(\mathrm{mol} \cdot \mathrm{kg}))$, and $I_{T}$ is an indicator function introduced such that $I_{T}=1$ for $T_{f}-\Delta_{f}<T<T_{f}+\Delta_{f}$, with $I_{T}=0$ for other temperatures.

The term $\dot{H}_{1}$ introduces a coupling between the heat transfer and the moisture transfer. For slow freezing or thawing, which al-

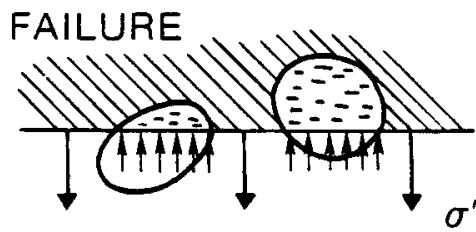

Fig. 4. Balance of stresses in the solid structure of the material and in the pores.

ways prevails in thicker cross sections, this term may probably be neglected. In that case, the temperature distribution problem becomes uncoupled, reduced to the usual heat conduction equation.

\section{Cracking Due to Freezing}

In rational analysis, the material failure due to freezing of water in the pores should be decided on the basis of the stresses produced in the solid structure of concrete and some form of strength or fracture criterion. For porous materials, this type of analysis is well-known from soil mechanics. The key concept is Terzaghi's effective stress, which represents the stress resultant in the solid structure of the material. Its volumetric component may be expressed as

$$
\sigma^{\prime}=\sigma+n p+n_{b} p_{b}
$$

in which $\sigma$ is the total volumetric stress in the material including the pores and bubbles, and $n$ and $n_{b}$ are the boundary porosities representing the cross-section areas over which pressures $p$ and $p_{b}$ act in the weakest cross sections through the material (Fig. 4). Without bubbles, $n<1$. For concrete, $n$ is apparently close to 1 (see Ref. 37). With the bubbles, it is required that $n+n_{b}=1$. Thus, one may safely use $n=1-n_{b}$, from which it follows that $n_{b}=1-\pi r_{b}^{2} / s_{b}^{2}$.

The material does not fail if, for each point of the cross section

$$
\sigma^{\prime} \leq f_{i}^{\prime}
$$

where $f_{t}^{\prime}$ is the tensile strength of concrete. The value of $f_{t}^{\prime}$ is not a constant but decreases with repeated stressing of the solid structure during cycles of freezing and thawing, just as during cycles of applied loads. When $\sigma=0$, which occurs in a load-free specimen without residual stresses, the criterion of no failure is

$$
n p+n_{b} p_{b} \leq f_{i}^{\prime}
$$

Normally, however, there are residual stresses due to freezing as well as drying, and further stresses are superimposed by loads applied on the structure. In the cross section of a compressed member, concrete can obviously sustain much higher pore pressures without any cracking than can concrete in a tensioned or load-free cross section. Therefore, it is necessary to calculate the stresses in concrete. For this purpose, the stress-strain law may be expressed as a relation of the strain to the effective stress rather than to the total stress in the material. For example, in the simplest case of elastic analysis. the stress-strain relations are $\sigma^{\prime}=3 K e$ and $s_{i j}=2 G e_{i j}$ where $K$ and $G$ are the bulk shear elastic moduli of concrete, and $s_{i j}$ are deviatoric stresses. The total stresses in the solid structure are expressed as $\sigma_{i j}=s_{i j}+\delta_{i j} \sigma$ where subscripts $i$ and $j$ refer to Cartesian components in coordinates $x_{i}, i=1,2,3$, and $\delta_{i j}$ is the Kronecker delta. The differential equations of equilibrium. on the other hand. must be expressed in terms of the total stresses rather than the effective stresses, i.e., $\sigma_{i j, j}=0$.

More realistically, one would need to consider nonlinear timedependent material behavior, which can be accomplished by finite-element analysis. The present formulation may serve as a basis for such a finite-element program. The effect of the rate of freezing on stress development should also be considered.

The problem of freeze-thaw durability is in practice often 
coupled with reinforcement corrosion. ${ }^{36,3 y}$ The cracking due to volume expansion of rust or to freeze-thaw cycles accelerates the moisture ingress into concrete.

\section{Conclusion}

While the equations governing the basic physical processes involved in freezing and thawing of concrete are known, a comprehensive mathematical model was unavailable and is developed in the present study. This model involves (1) formulation of the sorption isotherms of frozen concrete on the basis of the given isotherms of unfrozen concrete, (2) formulation of the diffusion of water, including both the macro- and microscopic diffusions through concrete as well as a microscopic (local) diffusion into air-entrained bubbles, (3) calculation of temperature distribution taking into account the latent heat of freezing, and finally (4) calculation of the stresses in the solid structure of concrete. This model makes it, in principle, feasible to determine cracking due to freezing on the basis of the stresses in the solid structure of concrete. Solution of the differential equations presented would require finite-element computer analysis. To make application of the mathematical model possible, it will be necessary to first calibrate it by carrying out various new types of tests, which would have to include freezing and thawing of specimens under load for various load levels, types of loads, cross-section sizes, and freezing rates.

\section{APPENDIX}

\section{Some Comments on Dilatometric Tests}

Freeze-thaw durability is often evaluated on the basis of cyclic dilatometric tests (Powers' test ASTM C671), although the standard ASTM test C666 does not use dilatometry. A typical dilatometric test is exhibited in Fig. Al. A noteworthy feature is the change of slope after freezing as well as a vertical jump at the time of freezing.

These properties may be explained by a simple composite model in which $\boldsymbol{n}$ denotes the volume fraction of freezable pores in concrete. The equilibrium of longitudinal normal stresses in the specimen requires that $\sigma=(1-n) \sigma_{c}+n \sigma_{i}$ in which $\sigma$ is the total stress, $\sigma_{c}$ and $\sigma_{i}$ are normal stresses in the solid structure of concrete and in ice, $E_{c}$ and $E_{i}$ are elastic moduli of concrete and of ice, $\alpha_{r}$ and $\alpha_{i}$ are linear coefficients of thermal expansion of concrete and ice, and $\varepsilon_{0}^{\prime}=q \varepsilon_{0}$ where $\varepsilon_{0}$ is the volume expansion of water on freezing and $q$ indicates a reduction in volume decrease due to the fact that pore volume $n$ may be filled by liquid water only partially or that part of the water may be expelled from these pores before it freezes. By algebraic rearrangement of the foregoing equation, one obtains for the strength after freezing the equation $\varepsilon=\bar{\alpha} T+\bar{\varepsilon}_{0}+\sigma / E$, in which $\bar{E}=(1-n) E_{c}+n E_{i}$. These two parameters are graphically shown in the schematic dilatometric diagram in Fig. Al $(B)$. From the preceding equations we obtain

$$
\begin{aligned}
& \bar{\alpha}=\frac{(1-n) E_{c} \alpha_{c}+n E_{i} \alpha_{i}}{\bar{E}} \\
& \bar{\varepsilon}_{0}=\frac{E_{i}}{\bar{E}} q n \varepsilon_{0}^{\prime}
\end{aligned}
$$

If the freezing temperature $T_{f}$ (Fig. $\left.A l(B)\right)$ is known, one could calculate from Eqs. $(\mathrm{A}-1 a)$ and $(\mathrm{A}-1 b)$ the values of $n$ and $q$.

According to the linear diffusion equation for heat conduction, the temperature difference between the middle and the surface of a slab at a constant cooling rate $\dot{T}$ may be estimated as $\Delta T=$ $T D^{2} \mathrm{C} \rho / 4 \mathrm{c}$ in which $C=753 \mathrm{~J} /\left({ }^{\circ} \mathrm{C} \cdot \mathrm{kg}\right)$ is the heat capacity of concrete, and $C_{0}=c / C$ is the heat diffusivity of concrete (about $0.004 \mathrm{~m}^{2} / \mathrm{h}$ ). For a typical cooling rate $\dot{T}=10^{\circ} \mathrm{C} / \mathrm{h}$ and thickness $=2.5 \mathrm{~cm}$, this temperature difference is found to be $\Delta T \approx$ $1^{\circ} \mathrm{C}$. This is rather small, which means that the internal stresses due to the delay from heat conduction are insignificant for the typical dilatometric tests. However, for thicker bodies the temperature difference can be quite significant; e.g., for thickness $D=10 \mathrm{~cm}$, one gets for the same cooling rate $\Delta T=16^{\circ} \mathrm{C}$. Thus, the durability reduction caused by nonuniform stresses due to heat conduction and diffusion can be determined only when comparisons are made with much larger specimens. The dilatometric tests represent only a small part of the experimental information which would be needed to calibrate the present model.

With regard to the offset of the curves in Fig. $\mathrm{Al}(A)$, it is of interest to estimate the temperature rise in concrete, $\Delta T_{f r}$, caused

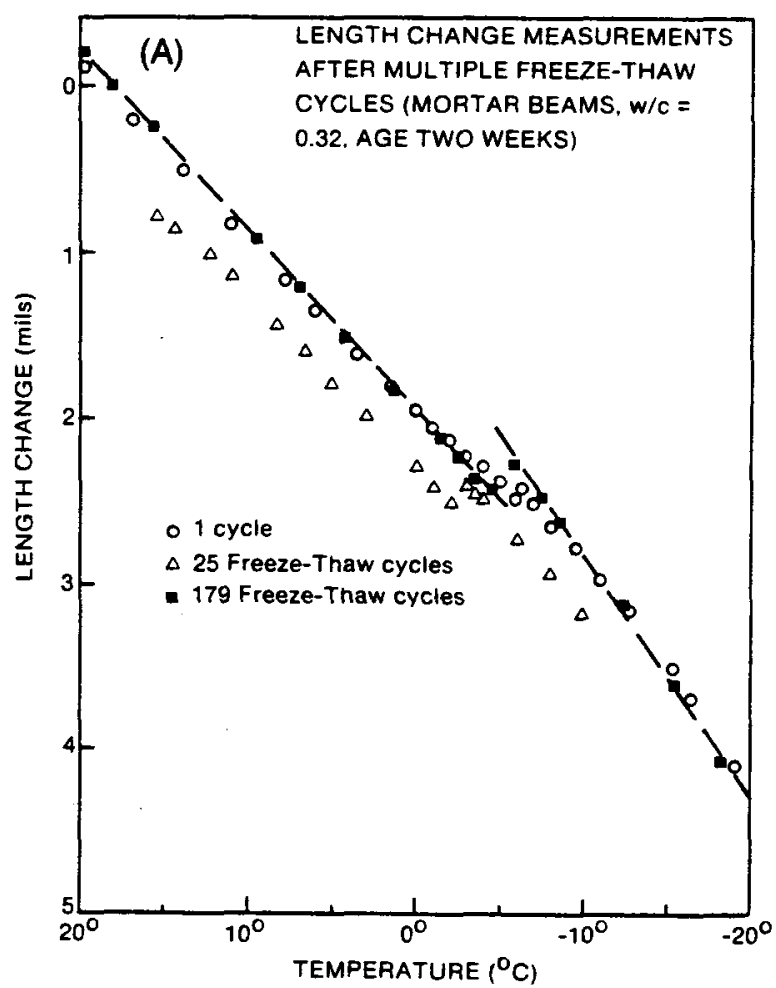

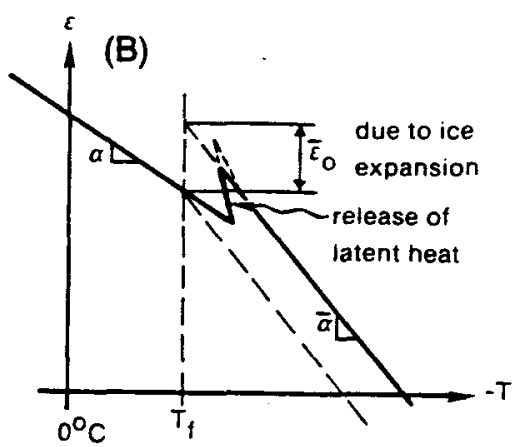

Fig. A1. Example of dilatometric tests. 
by the release of latent heat on freezing. We have $\Delta T_{f r}=C_{w} w /$ $C \rho$, in which for a typical concrete we may substitute $C_{w}=$ $333000 \mathrm{~J} /(\mathrm{mol} \cdot \mathrm{kg}), \rho=2300 \mathrm{~kg} / \mathrm{m}^{3}$, and $w=3.8 \mathrm{~mol} \cdot \mathrm{kg}$ where $w$ is the content of freezable water. Calculation then yields $\Delta T_{f r}=0.7^{\circ} \mathrm{C}$. Under adiabatic conditions, this would be manifested as an increase of temperature. However, when the environmental temperature is controlled, the temperature rise would have to gradually dissipate in an exponential fashion, as indicated by the dashed upward projection in Fig. $\mathrm{Al}(B)$. For this reason. $\Delta T_{f r}$ is not the major contribution to the offset of the straight lines in Fig. $A l(B)$.

\section{References}

'J. R. Blachere and J.E. Young, "The Freezing Point of Water in Porous Glass," J. Am. Ceram. Soc.. 55 [6] 306-308 (1972).

2P. D. Cady, "Mechanisms of Frost Destruction in Concrete": Ph.D. Thesis. Pennsyivania State University, University Park, PA. 1967. P. D. Cady, J. Mater. 4 [2] 294-311 (1969).

J. R. Dunn and P.P. Hudec. "The Influence of Clay on Water and Ice in Rock Pores." Physical Research Rept. No. RR 65-5. New York State Department of Public Works, New York. 1965

${ }^{4} \mathrm{G}$. Fagerlund. "The Significance of Critical Degrees of Saturation at Freezing of Porous and Brittle Materials": pp. 13-65 in Durability of Concrete, ACI SP-47, American Concrete Institute. Detroit. MI. 1975.

${ }^{5} \mathrm{G}$. Fagerlund, "Studies of the Destruction Mechanism at Freezing of Porous Material," Rept. No. 1:76, Swedish Cement and Concrete Research Institute, Stockholm. Sweden, 1976

${ }^{\circ} \mathrm{G}$. Fagerlund. "The Critical Degree of Saturation Method of Assessing the Freeze/Thaw Resistance of Concrete," Mater. Constr. (Paris). 10 [58] 217-29 1977.

G. Fagerlund. "Prediction of the Service Life of Concrete Exposed to Frost Action": pp. 249-76 in Studies on Concrete Technology. Swedish Cement and Concrete Research Institute, Stockhoim. Sweden, 1979.

${ }^{8}$ G. Fagerlund, "Service Life of Concrete Structure"; in Proceedings of the Conference on Contemporary European Concrete Research, Stockholm. Sweden, June 1981. Swedish Cement and Concrete Institute. Stockholm. Sweden.

9 J. C. Fisher, J. H. Holloman, and D. Turnbull, Science. 109. 168-69 (1949).

${ }^{10}$ R. A. Helmuth, "Capillary Size Restrictions on Ice Formation in Hardened Portland Cement Pastes," Paper V1-S2: pp. 855-69 in Proceedings of the Fourth International Symposium on Chemistry of Cement. Washington, DC, 1960, U.S National Bureau of Standards. Washington. DC.

"Z. Jacobi. "On ice Formation in Pure Undercooled Water" (in Ger.), Z. Naturforsch.. $A, 10,322-30$ (1955).

${ }_{12}$ V. P. Kubelka and R. Prokscha, "A New Method to Determine the Surface Tension of Crystais" (in Ger.), Kolloid-Z., 109. 79-85 (1944).

${ }^{13}$ B. R. Puri et al. "Freezing Point of Water Held in Porous Bodies at Different Vapor Pressures," Phys. Chem., 58 [4] 289-92 (1954).

i4 P. K. Mehta and O. E. Gjørv. "Properties of Portland Cement Concrete Containing Fly Ash and Condensed Silica-Fume." Cem. Concr. Res.. 12, 587-95 (1982).

is V. Moldovan, Additives in Concrete (Aditivi in Betoane). Ed. Technica, Bucuresti. Romania, 1978.

${ }^{16}$ A. M. Neville, Properties of Concrete. Pitman Publishing, London, UK, 1981

${ }^{17}$ T. C. Paver, "The Air Requirement of Frost Resistant Concrete," Proc. Highw. Res. Board, 29, 285 (1949).
${ }^{18}$ T. C. Powers, "Resistance of Concrete to Frost at Early Ages"; pp. 1-46 in Proceedings of the RILEM Symposium: Winter Concreting, Copenhagen, Denmark. 1956.

${ }^{19}$ T. C. Powers and R. A. Helmuth. "Theory of Volume Changes in Hardened Portland Cement Paste During Freezing," Proc. Highw. Res. Board, 32, 285 (1953)

${ }^{20}$ B. R. Puri et al., J. Phys. Chem., 58, 289-92 (1954).

"I B. R. Puri. D. C. Singh, and T. P. Myer, "Freezing Points of Liquids Adsorbed on Porous Solids," Trans. Faraday Soc., 53. 530-34 (1957).

${ }^{22} \mathrm{M}$. Volmer, Kinetics of Phase Formation (Kinetik der Phasenbildung). Theodor Steinkoff, Dresden, East Germany, 1939.

${ }^{23}$. Warris. "The Influence of Air-Entrainment on the Frost Resistance of Concrete," Rept. No. 36. Swedish Cement and Concrete Research Institute, Stockholm. Sweden. 1964.

${ }^{24} \mathrm{H}$. G. Wheat. "An Electrochemical Investigation of the Corrosion of Steel in Concrete"; Ph.D. Dissertation. University of Texas, Austin, TX, 1985.

${ }^{23}$ Z. P. Bazant, "Constitutive Equation for Concrete Creep and Shrinkage Based on Thermodynamics of Multiphase Systems," Mater. Constr. (Paris), 3, 3-36 (1970).

${ }^{25} \mathrm{Z}$. P. Bazant. "Thermodynamics of Hindered Adsorption with Application to Cement Paste and Concrete," Cem. Concr. Res.. 2, 1-16 (1972).

${ }^{27} Z$. P. Bażant. "Thermodynamics of Interacting Continua with Surfaces and Creep Analysis of Concrete Structures," Nucl. Eng. Des., 20. 477-505 (1972).

${ }^{20}$ Z. P. Batant, "Surface Diffusion Theory for the Drying Creep Effect in Portland Cement Paste and Concrete," J. Am. Ceram. Soc., 56, 235-41 (1973).

${ }^{29}$ RILEM Committee TC 69 , State-of-the-Art Report on "Mathematical Modeling of Creep and Shrinkage"; Ch. 1, pp. 43-78 in Preprints. 4th RILEM Internationa Symposium on Creep and Shrinkage of Concrete: Mathematical Modeling, Northwestem University, Evanston, IL. Edited by Z. P. Bażant. Northwestem University Press, Evanston. IL.

${ }^{30}$ A. W. Anderson, A Textbook of Physical Chemistry, 3d ed. Academic Press, Orlando. FL, 1986

${ }^{1}$ T. C. Powers, L. E. Copeland, and H. M. Mann. "Capillary Continuity and Discontinuity in Cernent Paste," J. Portland Cem. Assoc., Res. Dev. Lab.. 1 [2] 38-48 (1959)

${ }^{32}$ D. A. Rose, "Water Movement in Unsaturated Porous Materials," RILEM Bull. 29. 119-24 (1965).

${ }^{33}$ Z. P. Bažant. J. C. Chern, and W. Thonguthai, "Finite Element Program for Moisture and Heat Transfer in Heated Concrete," Nucl. Eng. Des., 68, 61-70 (1981).

${ }^{34}$ Z. P. Bažant and L. J. Najjar, "Nonlinear Water Diffusion in Nonsaturated Concrete." Mater. Constr. (Paris), 5, 3-20 (1972).

${ }^{39}$ Z. P. Bažant and W. Thonguthai. "Pore Pressure and Drying of Concrete at High Temperature," J. Eng. Mech. Div.. Am. Sac. Civ. Eng., 104. 1058-80 (1978).

${ }^{36} Z$. P. Bazant and W. Thonguthai, "Pore Pressure in Heated Concrete Walls," Mas. Concr. Res.. 31. 67-76 (1979).

M7. P. Bazant. "Pore Pressure. Uplift and Failure Analysis of Dams"; pp. 781808 in Proceedings, International Symposium on Criteria and Assumptions for Numerical Analysis of Dams. Department of Civil Engineering, University of Wales, Swansea, and British National Committee on Large Dams. Swansea, 1975.

${ }^{38}$ Z. P. Bażant. "Physical Model for Steel Corrosion in Concrete Sea Structures I. Theory, II. Application," J. Eng. Mech. Div., Am. Soc. Civ. Eng., 105, 1137-95 (1979).

${ }^{39}$ Z. P. Bažant. "Nonstationary Long-Time Processes Causing Loss of Serviceability": pp. 261-84 in Proceedings, IABSE (International Association for Bridge and Structural Engineering. Zürich) Colloquium on Computational Mechanics of Concrete Structures - Advances and Applications, Delft. The Netherlands, August 1987. International Association for Bridge and Structural Engineering, Zurich Switzerland, 1987. 